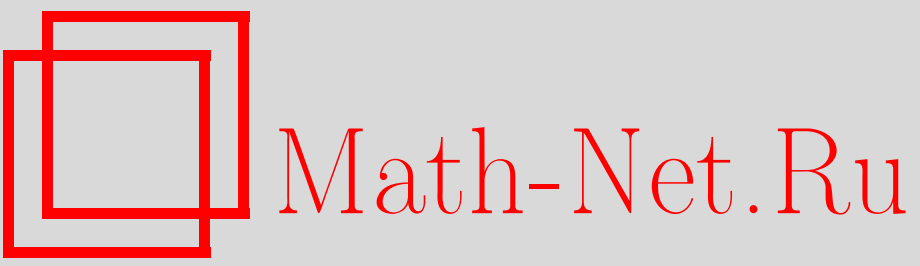

И. А. Александрова, С. Е. Степанов, И. И. Цыганок, От гармонических отображений к потокам Риччи на основе техники Бохнера, Итоги науки и техн. Сер. Соврем. мат. и ее прил. Темат. обз., 2019, том 169, 75-87

DOI: https://doi.org/10.36535/0233-6723-2019-169-75-87

Использование Общероссийского математического портала Math-Net.Ru подразумевает, что вы прочитали и согласны с пользовательским соглашением

http://www.mathnet.ru/rus/agreement

Параметры загрузки:

IP : 52.87 .193 .239

26 апреля 2023 г., 13:37:24 


\title{
ОТ ГАРМОНИЧЕСКИХ ОТОБРАЖЕНИЙ К ПОТОКАМ РИЧЧИ НА ОСНОВЕ ТЕХНИКИ БОХНЕРА
}

\author{
() 2019 г. И. А. АЛЕКСАНДРОВА, С. Е. СТЕПАНОВ, И. И. ЦЫГАНОК
}

\begin{abstract}
АннотАция. Статья посвящена изучению глобальных аспектов геометрии гармонических отображений, в частности, инфинитезимальных бесконечно малых гармонических преобразований, и применению этих отображений к теории солитонов Риччи. Результаты получены при помощи методов геометрического анализа, в частности, теорем Яу, Ли и Шона о связях между геометрией полного гладкого многообразия и глобальным поведением субгармонических функций на нем.
\end{abstract}

Ключевые слова: гармоническое отображение, поток Риччи, солитон Риччи, техника Бохнера, субгармоническая функция.

\section{FROM HARMONIC MAPPINGS TO RICCI FLOWS DUE TO THE BOCHNER TECHNIQUE}

\author{
(c) 2019 I. A. ALEKSANDROVA, S. E. STEPANOV, I. I. TSYGANOK
}

\begin{abstract}
The present paper is devoted to the study a global aspect of the geometry of harmonic mappings and, in particular, infinitesimal harmonic transformations, and represents the application of our results to the theory of Ricci solitons. These results will be obtained using the methods of Geometric analysis and, in particular, due to theorems of Yau, Li and Schoen on the connections between the geometry of a complete smooth manifold and the global behavior of its subharmonic functions.
\end{abstract}

Keywords and phrases: harmonic mapping, Ricci flow, Ricci soliton, Bochner technique, subharmonic function.

AMS Subject Classification: 53C20; 53C43; 53C44

1. Введение. Хорошо известно, что существует связь между гармоническими отображениями и потоками Риччи, устанавливаемая при помощи «трюка Детурка», который превращает поток Риччи в нелинейное параболическое уравнение (см. [15, с. 113-118]). С другой стороны, автомодельные решения потока Риччи представляют собой солитоны Риччи (см. [14, с. 22]). В то же время векторное поле, превращающее риманову метрику в солитон Риччи, является инфинитезимальным гармоническим преобразованием (доказательство см. в $[7,30]$ ). В связи с этим основной целью данной работы будет изучение функционально-теоретических аспектов глобальной дифференциальной геометрии гармонических отображений (см. $[17,23,29,35])$ и, в частности, инфинитезимальных гармонических преобразований (см. $[25,31])$ и солитонов Риччи (см. $[14,18])$, а также потоков Риччи (см. [15]). Структура статьи такова. В разделе 2 приведен краткий обзор основных фактов геометрии «в целом» гармонических отображений римановых многообразий. В частности, доказано, что классические теоремы о гармонических отображениях являются следствиями известных фактов теории субгармонических функций. Результаты раздела 3 получены 
по аналогии с результатами раздела 2. В свою очередь, результаты раздела 4 являются приложениями результатов раздела 3 . В заключение рассмотрены уравнения эволюции скалярной кривизны и тензора Риччи с точки зрения техники Бохнера.

Указанные темы удается связать воедино при помощи техники Бохнера и ее обобщенного варианта. Напомним, что классическая техника Бохнера - это аналитический метод получения теорем исчезновения для некоторых топологических и геометрических инвариантов на компактных (без края) римановых многообразиях при некоторых предположениях относительно кривизны (см. [8, 9,34] и [27, Chap. 9]). Доказательства таких теорем сводятся к применению приниипа максимума Бохнера и теоремы Грина (см. [9, с. 30-31]). В настоящей работе мы также используем обобщенный вариант техники Бохнера (см. [28]) и, в частности, прини,ип максимума Хопфа (см. [10]) и результаты Яу, Ли и Шена о связи между геометрией полного гладкого многообразия и глобальным поведением субгармонических функций на нем (см. $[24,36])$.

Утверждения, доказанные в этой работе, дополняют ранние результаты авторов (см. [3-5,31, 33]) и других исследователей (см. [13, 18, 32,34] и [2, с. 57]).

2. Гармонические отображения с точки зрения техники Бохнера. Этот раздел содержит краткий обзор основных фактов дифференциальной геометрии гармонических отображений между римановыми многообразиями (подробности см. в $[23,35])$. Тема гармонических отображений весьма обширна и имеет множество применений; для охвата всех ее аспектов, даже поверхностно, потребовалось бы много времени (см. [23, p. 417]). Мы осветим лишь некоторые вопросы, в частности, ключевую проблему об отсутствии гармонических отображений между заданными полными римановыми многообразиями. Для ответа на этот вопрос будут привлечены некоторые методы геометрического анализа (см. [27, Chap. 9], [28]). В частности, мы докажем новые версии двух известных теорем об исчезновении гармонических отображений из $[17,29]$. Отметим, что вопрос о доказательстве существования гармонических отображений изучался в [23].

Пусть $(M, g)$ и $(\bar{M}, \bar{g})$ - полные римановы многообразия размерностей $n$ и $\bar{n}$, соответственно. Плотностъю энергии гладкого отображения $f:(M, g) \rightarrow(\bar{M}, \bar{g})$ назовем такую неотрицательную скалярную функцию $e(f): M \rightarrow \mathbb{R}$, что $e(f)=\frac{1}{2}\left\|f_{*}\right\|^{2}$, где $\left\|f_{*}\right\|^{2}$ - квадрат нормы дифференциала $f_{*}$, вычисленный относительно метрики $\tilde{g}$ на векторном расслоении $T^{*} M \otimes f^{*} T \bar{M}$, индуцированной метриками $g$ и $\bar{g}$ (см. [17]).

Известно, что отображение $f:(M, g) \rightarrow(\bar{M}, \bar{g})$ является гармоническим тогда и только тогда, когда оно удовлетворяет уранению Эйлера-Лагранжа

$$
\operatorname{trace}_{g}\left(\tilde{\nabla} f_{*}\right)=0,
$$

где $\tilde{\nabla}=\nabla \oplus \bar{\nabla}$ - каноническая связность в векторном расслоении $T^{*} M \otimes f^{*} T \bar{M}$ (см. [15, с. 117] и $[17,23])$. Кроме того, для гармонического отображения $f$ стандартными вычислениями можно убедиться (см. [17, с. 123]) в справедливости формулы

$$
\Delta e(f)=\left\|\tilde{\nabla} f_{*}\right\|^{2}+Q(f)
$$

где $\Delta:=\operatorname{div} \nabla-$ оператор Лапласа-Бельтрами, а скалярная функция $Q(f)$ имеет вид

$$
Q(f)=g\left(\operatorname{Ric}, f^{*} \bar{g}\right)-\operatorname{trace}_{g}\left(\operatorname{trace}_{g}\left(f^{*} \bar{R}\right)\right),
$$

где $\bar{R}$ - тензор римановой кривизны многообразия $(\bar{M}, \bar{g})$ и $\operatorname{Ric}-$ тензор Риччи многообразия $(M, g)$.

В [32] было найдено условие, когда $Q(f)$ является квазиположительной скалярной функцией на связной открытой области $U \subset M$, т.е. $Q(f)$ неотрицательна всюду в $U$ и $Q(f)$ положительна

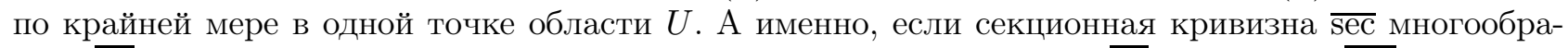
зия $(\bar{M}, \bar{g})$ неотрицательна в произвольной точке области $f(U) \subset \bar{M}$ и $\operatorname{Ric} \geqslant f^{*} \overline{\operatorname{Ric}}$ в каждой точке области $U$ для тензора Риччи $\overline{\operatorname{Ric}}$ многообразия $(\bar{M}, \bar{g})$, то $Q(f) \geqslant 0$. Более того, если существует точка области $U$, в которой $\mathrm{Ric}>f^{*} \overline{\mathrm{Ric}}$, то $Q(f)>0$ в этой точке. В этом случае $Q(f)$ также является квазиположительной скалярной функцией, определенной на $U$. Следовательно, согласно $(2.2)$, функция плотности энергии $e(f)$ удовлетворяет неравенству $\Delta e(f) \geqslant 0$ 
в каждой точке области $U$ и, стало быть, является субгармонической функиией. Из предположения, что функция плотности энергии $e(f)$ достигает локального максимума в некоторой точке $x \in U$, следует, что $e(f)$ принимает постоянное значение $C$ в $U$ согласно приниипу максимума Xonøa (см. [9, Theorem 2.1] и [10, Theorem 1]). Если $C>0$, то $\nabla f$ нигде не обращается в нуль. Таком образом, в точке, где $Q(f)$ положительна, левая часть (2.2) равна нулю, в то время как правая часть положительна. Полученное противоречие показывает, что $C=0$ и, следовательно, $f$ постоянно в $U$. Итак, мы доказали следующую лемму.

Лемма 2.1. Пусть $f:(M, g) \rightarrow(\bar{M}, \bar{g})$ - гармоническое отображение, плотность энергии которого е $(f)$ имеет локальный максимум в некоторой точке $x$ связной открытой области $U \subset M$. Кроме того, предположим, что секиионная кривизна $\overline{\sec }$ многообразия $(\bar{M}, \bar{g})$ неотрицательна в произвольной точке области $f(U) \subset \bar{M},(M, g)$ имеет тензор Риччи Ric, удовлетворяющий условию $\mathrm{Ric} \geqslant f^{*} \overline{\mathrm{Ric}}$ в каждой точке области $U$, и существует хотя бъ одна точка области $U$, в которой $\mathrm{Ric}>f^{*} \overline{\mathrm{Ric}}$. Тогда отображение $f$ постоянно в области $U$.

Используя лемму 2.1 и принцип максимума Бохнера (см. [9, Theorem 2.2]), получим теорему, доказанную авторами в [32].

Теорема 2.2. Пусть $f:(M, g) \rightarrow(\bar{M}, \bar{g})$ - гармоническое отображсние между римановыми

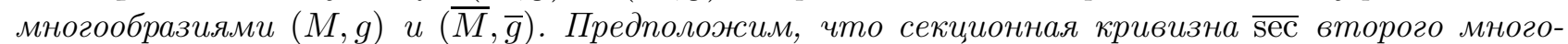
образия $(\bar{M}, \bar{g})$ неотрицательна в кажсдой точке области $f(M)$, а первое многообразие $(M, g)$ компактно и его тензор Риччи удовлетворяет условию $\mathrm{Ric} \geqslant f^{*} \overline{\mathrm{Ric}}$. Тогда $f$ является вполне геодезическим отображением с постоянной плотностъю энергии е(f). Кроме того, если найдется хотя би одна точка многообразия $M$, в которой $\mathrm{Ric}>f^{*} \overline{\mathrm{Ric}}$, то $f-$ постоянное отображсние.

Замечание 2.3. Напомним, что в [17] была доказана следующая знаменитая теорема исчезновения для гармонических отображений: если $f:(M, g) \rightarrow(\bar{M}, \bar{g})-$ произвольное гармоническое отображение между компактным римановым многообразием $(M, g)$ с тензором Риччи Ric $\geqslant 0$

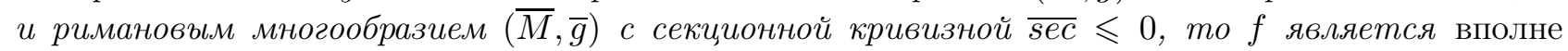
геодезическим $и$ имеет постоянную плотность энергии $e(f)$. Кроме того, если найдется хотя бы одна точка многообразия $M$, в которой кривизна Риччи положительна, $\mathrm{Ric}>0$, то любое гармоническое отображение $f:(M, g) \rightarrow(\bar{M}, \bar{g})$ постоянно.

Ли и Шен доказали (см. [24]), что на любом полном римановом многообразии $(M, g)$ с неотрицательным тензором Риччи не существует непостоянных неотрицательных $L^{p}$-интегрируемых $(0<p<\infty)$ субгармонических функций $u$. Другими словами, если предположить, что Ric $\geqslant 0$ и

$$
\int_{M}(\|u\|)^{p} d \operatorname{Vol}_{g}<\infty
$$

для некоторого полного риманова многообразия $(M, g)$, то $u=C$, где $C$ - некоторая константа. В этом случае

$$
C^{p} \int_{M} d \operatorname{Vol}_{g}<\infty .
$$

Если $C>0$, то $u$ нигде не обращается в нуль и объем многообразия $(M, g)$ конечен. Кроме того, известно (см. [36]), что любое полное некомпактное риманово многообразие $(M, g)$ с неотрицательным тензором Риччи имеет бесконечный объем. Это противоречие показывает, что $C=0$ и, следовательно, $u \equiv 0$. Итак, мы можем сформулировать следующую лемму.

Лемма 2.4. На полном некомпактном римановом многообразии $(M, g)$ с неотрицательным тензором Риччи не существует ненулевых неотрицательных $L^{p}$-интегрируемых $(0<p<\infty)$ субгармонических функиий. 
Пусть $(M, g)$ - полное некомпактное риманово многообразие. Для данного гладкого отображения $f:(M, g) \rightarrow(\bar{M}, \bar{g})$ определим его энергию следующим образом (см. [17,29]):

$$
E(f)=\int_{M} e(f) d \operatorname{Vol}_{g} .
$$

Для гладкого отображения $f:(M, g) \rightarrow(\bar{M}, \bar{g})$ полного риманова многообразия $(M, g)$ энергия $E(f)$ может оказаться бесконечной или конечной. В частности, $E(f)<+\infty$ для компактного риманова многообразия $(M, g)$ (см. [29]).

В то же время мы можем сформулировать альтернативную теорему для гармонических отображений из полного риманова многообразия в риманово многообразие с неотрицательной секционной кривизной (см. [29] и [35, с. 25]). В этом случае для доказательства мы также можем использовать лемму 2.1 о ненулевых неотрицательных $L^{p}$-интегрируемых $(0<p<\infty)$ субгармонических функциях на полном римановом многообразии с неотрицательной кривизной Риччи. Именно, справедлива следующая теорема.

Теорема 2.5. Пусть $f:(M, g) \rightarrow(\bar{M}, \bar{g})$ - гармоническое отобраэсение с конечной энергией. Eсли $(M, g)$ - полное некомпактное риманово многообразие с тензором Риччи Ric $\geqslant f * \overline{\mathrm{Ric}}$, где $\overline{\mathrm{Ric}}$ - тензор Риччи многообразия $(\bar{M}, \bar{g}), u(\bar{M}, \bar{g})$ - риманово многообразие, секционная кривизна $\overline{\mathrm{sec}}$ которого неотрицательна в кажндой точке области $f(M)$, то $f$-постоянное отображение.

Замечание 2.6. Напомним следующую известную теорему исчезновения что Яу-Шена (см. [29]): гармоническое отображение с конечной энергией $E(f)$ из полного некомпактного многообразия $(M, g)$ с тензором Риччи Ric $\geqslant 0$ в компактное многообразие $(\bar{M}, \bar{g})$ с секционной кривизной $\overline{\mathrm{sec}} \leqslant 0$ гомотопно постоянному отображению.

3. Инфинитезимальные гармонические преобразования с точки зрения теории функций. Основные результаты этого раздела получены как аналоги результатов предыдущего раздела.

Векторное поле $\xi$ на полном римановом многообразии $(M, g)$ называется инфинитезимальным гармоническим преобразованием (см. [25]), если $\xi$ порожает поток, являющийся локальной однопараметрической группой гармонических преобразований $(t, x) \in \mathbb{R} \times M \rightarrow \phi_{t}(x) \in M$ (другими словами, локальных гармонических диффеоморфизмов). Аналитическая характеристика таких векторных полей имеет вид

$$
\operatorname{trace}_{g}\left(L_{\xi} \nabla\right)=0
$$

где $L_{\xi}$ - производная Ли в направлении поля $\xi$ (см. $\left.[25,31]\right)$. Эта формула аналогична формуле (2.1). Кроме того, в [31] было доказано, что векторное поле $\xi$ является инфинитезимальным гармоническим преобразованием тогда и только тогда, когда

$$
\tilde{\Delta} \theta=2 \operatorname{Ric}(\xi, \cdot)
$$

для 1-формы, соответствующей полю $\xi$ в силу двойственности, определенной метрикой $g$, и $л a-$ пласианом Ходжа-де Рама $\tilde{\Delta}$ (см. [8, с. 158]).

В соответствии с теорией гармонических отображений (см. [17]) определим плотность энергии потока на $(M, g)$, порожденного инфинитезимальным гармоническим преобразованием $\xi$, как скалярную функцию $e(\xi)=\frac{1}{2}\|\xi\|^{2}$, где $\|\xi\|^{2}=g(\xi, \xi)$. Тогда оператор Лапласа-Бельтрами $\Delta e(\xi)$ от плотности энергии $e(\xi)$ инфинитезимального гармонического преобразования $\xi$ имеет вид

$$
\Delta e(\xi)=\|\nabla \xi\|^{2}-\operatorname{Ric}(\xi, \xi)
$$

(см. $[3,5,30])$. Формула $(3.1)$ является аналогом формулы $(2.2)$ для плотности энергии $e(f)$ гармонического отображения $f$. Справедлива следующая теорема.

Теорема 3.1. Пусть $(M, g)$ - риманово многообразие и $U \subset M-$ связная открытая область. Если плотность энергии потока $е(\xi)$, порожденного инфинитезимальным гармоническим преобразованием $\xi$, имеет локальный максимум в некоторой точке области $U$ и кривизна Риччи многообразия $(M, g)$ квазиотрицательна в $U$, то $\xi \equiv 0$ всюду в $U$. 
Доказательство. Пусть кривизна Риччи многообразия $(M, g)$ квазиотрицательна всюду в связной открытой области $U \subset M$; тогда согласно (3.8) функция плотности энергии $e(\xi)$ удовлетворяет неравенству $e(\xi) \geqslant 0$. Это означает, что $e(\xi)$ - субгармоническая функция. Предположим теперь, что функция плотности энергии $e(\xi)$ достигает локального максимума в некоторой точке $x \in U$; тогда согласно принципу максимума Хопфа функция $e(\xi)$ постоянна в области $U, e(\xi)=C$ (см. [9, Theorem 2.1], [10, Theorem 1]). Если $C>0$, то $\xi$ нигде не обращается в нуль. Таким образом, в точке, где Ric отрицательна, левая часть уравнения (3.1) равна нулю, в то время как правая часть положительна. Из полученного противоречия следует, что $C=0$ и, следовательно, $\xi \equiv 0$ всюду в области $U$.

Замечание 3.2. Теорема 3.1 является прямым обобщением теоремы 4.3 из монографии Кобаяси о группах преобразований (см. [2, с. 57]) и теоремы Ву о киллинговом векторном поле, длина которого достигает локального максимума (см. [34]).

В качестве аналога теоремы 2.2 сформулируем следующую теорему, которую можно доказать при помощи приниипа максимума Бохнера (см. [9, Theorem 2.2]).

Теорема 3.3. Компактное риманово многообразие $(M, g)$ с квазиотрицательной кривизной Риччи не допускает ненулевых инфинитезимальных гармонических преобразований.

Далее, напомним (см. [1, с. 2]), что кинетической энергией $E(\xi)$ потока на многообразии $(M, g)$, порожденного векторным полем $\xi$, называется величина

$$
E(\xi)=\int_{M} e(\xi) d \operatorname{Vol}_{g} .
$$

Замечание 3.4. Это определение согласовано с теорией гармонических отображений в случае инфинитезимальных гармонических преобразований. Энергия $E(\xi)$ может быть конечной или бесконечной. Например, $E(\xi)<+\infty$ для гладкого полного векторного поля $\xi$ на компактном римановом многообразии $(M, g)$.

Следующее утверждение является аналогом теоремы 2.5 .

Теорема 3.5. Пусть $(M, g)$ - полное риманово многообразие с неположительной кривизной Риччи. Тогда каждое инфинитезимальное гармоническое преобразование с конечной кинетической энергией параллельно. Кроме того, если объем многообразия $(M, g)$ бесконечен или кривизна Риччи отрицательна в некоторой точке многообразия $M$, то любое инфинитезимальное гармоническое преобразование является тождественно нулевым на $(M, g)$.

Доказательство. Для доказательства используем известное второе неравенство Като (см. [8, c. 380]):

$$
-\|\xi\| \Delta\|\xi\| \leqslant g(\bar{\Delta} \theta, \theta)
$$

где $\bar{\Delta}:=-\operatorname{trace}_{g} \nabla \circ \nabla-$ грубый лапласиан и $\theta-1$-форма, соответствующая полю $\xi$ в силу двойственности, задаваемой метрикой $g$. Известно, что грубый лапласиан удовлетворяет формуле Вейтценбека (см. [2, с. 44], [8, с. 378]):

$$
\bar{\Delta} \theta=\tilde{\Delta} \theta-S \xi
$$

где $S$ - оператор Риччи, определенный равенством $g(S X, Y)=\operatorname{Ric}(X, Y)$, где $X$ и $Y$ - произвольные касательные векторные поля. Следовательно, второе неравенство Като можно переписать в виде

$$
2 \sqrt{e(\xi)} \Delta \sqrt{e(\xi)} \geqslant-g(\tilde{\Delta} \theta, \theta)+\operatorname{Ric}(\xi, \xi),
$$

где $\|\xi\|=\sqrt{2 e(\xi)}$. С другой стороны, в [5,31] доказано, что $\xi$ является инфинитезимальным гармоническим преобразованием на многообразии $(M, g)$ тогда и только тогда, когда $\tilde{\Delta} \xi=2 S \xi$. Таким образом, из (3.2) получаем следующее уравнение:

$$
\sqrt{e(\xi)} \Delta \sqrt{e(\xi)}=-\frac{1}{2} \operatorname{Ric}(\xi, \xi) .
$$


Предположим, что тензор Риччи Ric неположителен. В [36, с. 664] и [37] было показано, что любая неотрицательная гладкая функция $u$, определенная на полном римановом многообразии $(M, g)$ и удовлетворяющая условиям

$$
u \Delta u \geqslant 0, \quad \int_{M} u^{p} d \operatorname{Vol}_{g}<+\infty \quad \forall p \neq 1,
$$

постоянна. В частности, если объем многообразия $(M, g)$ бесконечен и $u=$ const, то $u=0$. Следовательно, если гладкое многообразие $(M, g)$ полно и

$$
E(\xi)=\int_{M} e(\xi) d \operatorname{Vol}_{g}<+\infty,
$$

то функция $\sqrt{e(\xi)}$ постоянна. В то же время из (3.4) следует, что объем многообразия $(M, g)$ конечен. Таким образом, из (3.2) получаем, что $\nabla \xi=0$. С другой стороны, если предположить, что $\operatorname{Ric}_{x}<0$ в некоторой точке $x \in M$, то это неравенство противоречит уравнению (3.1). Теорема доказана.

Замечание 3.6. Если $\xi$ - ненулевое векторное поле, для которого $\operatorname{Ric}(\xi, \xi) \leqslant 0$, то из уравнения (3.3) вытекает неравенство $\Delta \sqrt{e(\xi)} \geqslant 0$. Это означает, что $\sqrt{e(\xi)}$ - субгармоническая функция. Следовательно, приведенный выше результат является аналогом теоремы 2.5 .

Следствие 3.7. Пусть $(M, g)$ - связное полное некомпактное риманово многообразие размерности $n \geqslant 2$ с неприводимой группой голономи $\operatorname{Hol}(g)$ и неположительной кривизной Риччи. Тогда любое инфинитезимальное гармоническое преобразование на $(M, g)$ с конечной кинетической энергией тождественно равно нулю всюду на $(M, g)$.

Доказательство. Согласно теореме 3.5 инфинитезимальное гармоническое преобразование $\xi$ на связном полном некомпактном римановом многообразии $(M, g)$ с неположительной кривизной Риччи, для которого $E(\xi)<+\infty$, параллельно. В предположении, что группа голономии $\operatorname{Hol}(g)$ неприводима, это означает, что $\xi \equiv 0$.

Примером полного гладкого многообразия $(M, g)$ с неположительной кривизной Риччи может служить известное многообразие Картана-Адамара - односвязное полное риманово многообразие неположительной секционной кривизны (см. [28, с. 90]).

Следствие 3.8. Пусть $(M, g)$ - многообразие Картана-Адамара размерности $n \geqslant 2$, имеющее бесконечный обгем. Тогда любое инфинитезимальное гармоническое преобразование на многообразии $(M, g)$ с конечной кинетической энергией тождественно равно нулю всюду на $(M, g)$.

Замечание 3.9. Другие свойства инфинитезимальных гармонических преобразований можно найти в $[2-5,13,30,31]$. В частности, в [31] доказано, что множество всех инфинитезимальных гармонических преобразований на компактном римановом многообразии $(M, g)$ является конечномерным векторным пространством над $\mathbb{R}$. Кроме того, алгебра Ли $i(M)$ инфинитезимальных изометрических преобразований является подпространством этого векторного пространства (см. [31]). Напомним, что инфинитезимальное изометрическое преобразование (инфинитезимальная изометрия), или векторное поле Киллинга $X$ на $(M, g)$ определяется уравнением $L_{X} g=0$. Известно, что на $n$-мерном римановом многообразии $(M, g)$ постоянной кривизны $\operatorname{dim} i(M)=n(n+1) / 2$ (см., например, [2, с. 46-47]). Следовательно, размерность векторного пространства инфинитезимальных гармонических преобразований на $n$-мерном римановом многообразии $(M, g)$ постоянной кривизны не может быть меньше $n(n+1) / 2$. Отметим, что данный результат носит локальный характер. 
Следующее утверждение об инфинитезимальных изометрических преобразованиях хорошо известно (см. $[9$, с. 57$]$ и $[2$, с. 44$])$ : инфинитезимальная изометрия $\xi$ удовлетворяет дифференциальным уравнениям

$$
\begin{gathered}
\tilde{\Delta} \theta=2 \operatorname{Ric}(\xi, \cdot) ; \\
\operatorname{div} \xi=0,
\end{gathered}
$$

где $\theta$-1-форма, соответствующая полю $\xi$ в силу двойственности, задаваемой метрикой $g$. Обратно, если многообразие $(M, g)$ компактно и поле $\xi$ удовлетворяет уравнениям (3.5) и (3.6), то $\xi$ - инфинитезимальная изометрия.

Уравнение (3.6) более жестко, нежели требуется для приведенного выше утверждения. Сформулируем и докажем альтернативную версию этого утверждения.

Теорема 3.10. Пусть $(M, g)$ - риманово многообразие и $\xi$-векторное поле на $(M, g)$. Если поле $\xi$ является инфинитезимальной изометрией, то оно удовлетворяет следующим условиям: $\xi$-инфинитезимальное гармоническое преобразование и

$$
L_{\xi} \operatorname{div} \xi \geqslant 0 .
$$

Обратно, если $(M, g)$ компактно и $\xi$ удовлетворяет двум приведенным выше условиям, то $\xi-$ инфинитезимальная изометрия.

Доказательство. Мы уже показали, что если $\xi$-инфинитезимальное гармоническое преобразование, то оно удовлетворяет (3.5). Кроме того, если $\xi$ является инфинитезимальной изометрией, то выполняется уравнение $\operatorname{div} \xi=0$. Отсюда следует (3.7). Чтобы доказать обратное утверждение, предположим, что многообразие $M$ компактно и ориентируемо (если $M$ неориентируемо, то рассмотрим его ориентируемое двойное накрытие). Рассмотрим векторное поле $X=(\operatorname{div} \xi) \xi$ для произвольного инфинитезимального гармонического преобразования $\xi$ на $(M, g)$. Дивергенция $\operatorname{div} X$ имеет вид

$$
\operatorname{div} X=L_{\xi}(\operatorname{div} \xi)+(\operatorname{div} \xi)^{2} .
$$

Применяя классическую теорему Грина

$$
\int_{M} \operatorname{div} X d \operatorname{Vol}_{g}=0
$$

к полю $X=(\operatorname{div} \xi) \xi$, получим интегральную формулу

$$
\int_{M}\left(L_{\xi}(\operatorname{div} \xi)+(\operatorname{div} \xi)^{2}\right) d \operatorname{Vol}_{g}=0
$$

где $d \mathrm{Vol}_{g}$ - каноническая мера (или, иначе, элемент объема), ассоциированная с метрикой $g$. Если неравенство $L_{\xi}(\operatorname{div} \xi) \geqslant 0$ выполняется в точках многообразия $(M, g)$, то из $(3.9)$ заключаем, что $\operatorname{div} \xi=0$. Для завершения доказательства остается сослаться на теорему 3.10.

Замечание 3.11. Можно определить дивергенцию векторного поля при помощи гидродинамического подхода. Именно, дивергенцией гладкого векторного поля $\xi$ на многообразии $(M, g)$ называется скалярная функция, определенная соотношением

$$
(\operatorname{div} \xi) d \operatorname{Vol}_{g}=L_{\xi}\left(d \operatorname{Vol}_{g}\right)
$$

(см. $[2$, с. 6] и $[26$, с. 195]). Формула (3.10) описывает изменение формы объема под действием потока векторного поля. На основании этого факта функция $\operatorname{div} \xi$ была названа в [26, с. 195] логарифмической скоростью обгемного расширения вдоль потока, порожденного векторным полем $\xi$. Следовательно, $L_{\xi} \operatorname{div} \xi$ является мерой ускорения оббемного расширения, т.е. ускорения изменения элемента объема $d \mathrm{Vol}_{g}$ вдоль траекторий потока с вектором скорости $\xi$. В частности, условие $L_{\xi} \operatorname{div} \xi \geqslant 0$ означает, что $d \mathrm{Vol}_{g}$ - неубывающая функция вдоль траекторий этого потока.

Замечание 3.12. Из равенства $L_{\xi} g=0$ вытекает также условие инвариантности для тензора Риччи: $L_{\xi} \operatorname{Ric}=0$. Имеются работы по общей теории относительности (см., например, $\left.[16,20]\right)$, где вместо условия $L_{\xi} \mathrm{Ric}=0$ изучается «ослабленное условие» вида $\operatorname{trace}_{g}\left(L_{\xi} \mathrm{Ric}\right)=0$. 
Интересно отметить, что в случае компактного риманова многообразия $(M, g)$ добавление условия $L_{\xi}$ Ric $\geqslant 0$ (или $\leqslant 0$ ) к уравнению $(3.1)$ приводит к тому, что инфинитезимальное гармоническое преобразование $\xi$ становится в действительности инфинитезимальной изометрией. А именно, справедлива следующая теорема.

Теорема 3.13. Пусть $(M, g)$ - риманово многообразие и $\xi$-векторное поле на $(M, g)$. Если $\xi$-инфинитезимальная изометрия, то $\xi$ является инфинитезимальным гармоническим преобразованием и

$$
\operatorname{trace}_{g}\left(L_{\xi} \text { Ric }\right) \geqslant 0(\leqslant 0) .
$$

Обратно, если многообразие $(M, g)$ компактно и $\xi$ удовлетворяет сформулированным выше двух

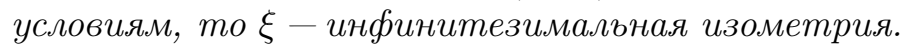

Доказательство. Выше было показано, что инфинитезимальное гармоническое преобразование $\xi$ удовлетворяет (3.5), т.е. первому условию теоремы. Кроме того, если $\xi$-инфинитезимальная изометрия, то выполняется уравнение $L_{\xi} \mathrm{Ric}=0$. Чтобы доказать обратное утверждение, предположим, что $M$ компактно. Инфинитезимальное гармоническое преобразование $\xi$ удовлетворяет дифференциальному уравнению

$$
\Delta \operatorname{div} \xi=\operatorname{trace}_{g}\left(L_{\xi} \operatorname{Ric}\right)
$$

(см. [6]). Используя принцип максимума Бохнера (см. [9, Theorem 2.2]), заключаем, что div $\xi=$ const. С другой стороны, имеем

$$
\int_{M} \operatorname{div} \xi d \operatorname{Vol}_{g}=0 .
$$

Следовательно, $\operatorname{div} \xi=0$, так что $\xi$-инфинитезимальное преобразование (см. теорему 3.10). Теорема 3.13 доказана.

Замечание 3.14. Теоремы 3.10 и 3.13 представляют собой альтернативные варианты классической теоремы об инфинитезимальных изометрических преобразованиях (см., например, $[9$, с. 57$]$ и $[2$, с. 44$])$.

4. Солитоны Риччи с точки зрения инфинитезимальных гармонических преобразований. Этот раздел посвящен приложениям результатов, полученных в разделе 3 , к теории солитонов Риччи.

Пусть $g$ - фиксированная риманова метрика на гладком многообразии $M$. Рассмотрим однопараметрическое семейство диффеоморфизмов $(t, x) \in \mathbb{R} \times M \rightarrow \phi_{t}(x) \in M$, порожденных гладким векторным полем $\xi$ на $M$. Эволюционирующая метрика $g(t)=\sigma(t) \phi_{t}^{*}(x) g(0)$, где $\sigma(t)$ - положительная скалярная функция, удовлетворяющая условию $\sigma(0)=1$, и $g(0)=g$, называется солитоном Риччи, если метрика $g$ является решением следующей нелинейной нестационарной системы уравнений с частными производными:

$$
-2 \text { Ric }=L_{\xi} g+2 \lambda g,
$$

где Ric-тензор Риччи метрики $g, L_{\xi} g-n$ роизводная Ли тензора $g$ вдоль векторного поля $\xi$ и $\lambda$-константа (см., например, [14, с. 22]). Будем обозначать солитон Риччи следующим образом: $(g, \xi, \lambda)$. В случаях $\lambda=0, \lambda<0$ и $\lambda>0$ соответственно солитон Риччи называется стационарным, сжимающимся или растягивающимся. Кроме того, солитон Риччи называется эйнштейновским, если $L_{\xi} g=0$, и тривиальнъм, если $\xi \equiv 0$.

В [7] была доказана следующая теорема.

Теорема 4.1. Векторное поле $\xi$ произвольного солитона Риччи $(g, \xi, \lambda)$ на гладком многообразии $M$ является инфинитезимальным гармоническим преобразованием на римановом многообразии $(M, g)$.

Таким образом, из теорем 3.1 и 4.1 получаем следующее утверждение. 
Следствие 4.2. Пусть $(g, \xi, \lambda)$ - солитон Риччи на гладком многообразии M. Если тензор Риччи Ric метрики g квазиотрицателен в связной открытой области $U \subset M$ и плотность энергии потока, порожденного векторным полем $\xi$, имеет локалъный максимум в некоторой точке области $U$, то $(g, \xi, \lambda)$ - растягивающийся солитон Риччи.

Доказательство. Пусть $(g, \xi, \lambda)$ - солитон Риччи на гладком многообразии $M$. Тогда его векторное поле $\xi$ является инфинитезимальным гармоническим преобразованием на римановом многообразии $(M, g)$. Кроме того, если тензор Риччи Ric метрики $g$ квазиотрицателен в связной открытой области $U \subset M$ и плотность энергии $e(\xi)$ потока, порожденного полем $\xi$, имеет локальный максимум в некоторой точке $x$ области $U$, то согласно теореме 3.1 имеем $\xi \equiv 0$ в каждой точке $y \in U$. В этом случае из (4.1) получаем равенство $\mathrm{Ric}_{y}=-\lambda g_{y}$; следовательно, $\lambda>0$.

Замечание 4.3. Из следствия 3.7 заключаем, что солитон Риччи $(g, \xi, \lambda)$ с квазиотрицательной кривизной Риччи метрики $g$ тривиален на компактном гладком многообразии $M$.

Из теоремы 3.3 получаем следующее утверждение.

Следствие 4.4. Пусть $(g, \xi, \lambda)$ - солитон Риччи на компактном гладком многообразии $M$. Если элемент обгема $d \mathrm{Vol}_{g}$ (соответственно, скалярная кривизна $s$ метрики $g$ ) является неубывающей (соответственно, невозрастающей) функиией вдоль траекторий потока с вектором скорости $\xi$, то солитон Риччи $(g, \xi, \lambda)$ тривиален.

Доказательство. Рассмотрим солитон Риччи $(g, \xi, \lambda)$ на компактном гладком многообразии $M$. Из (4.1) для скалярной кривизны $s=\operatorname{trace}_{g} \operatorname{Ric}$ получим $\operatorname{div} \xi=-(s+n \lambda)$; тогда ускорение объемного расширения потока, порожденного векторным полем $\xi$ солитона Риччи $(g, \xi, \lambda)$, имеет вид

$$
L_{\xi}(\operatorname{div} \xi)=-L_{\xi} s
$$

Следовательно, ускорение изменения элемента объема $d \mathrm{Vol}_{g}$ вдоль траекторий потока с вектором скорости $\xi$ равно $-L_{\xi} s$. В частности, условие $L_{\xi} s \leqslant 0$ означает, что $d \mathrm{Vol}_{g}$ - неубывающая функция вдоль траекторий потока. С другой стороны, если $L_{\xi} s \leqslant 0$, то из теоремы 3.13 получаем, что $\xi$ - инфинитезимальная изометрия, т.е. $L_{\xi} g=0$. Произвольный солитон Риччи $(g, \xi, \lambda)$ на компактном гладком многообразии $M$ является градиентным, т.е. $\theta=\operatorname{grad} u$, где $u \in C^{\infty} M-$ некоторая функция (см. [18]). Следовательно, условие $L_{\xi} g=0$ превращается в $\nabla \nabla u=0$, откуда вытекает уравнение $\Delta u=0$, т.е. $u$ - гармоническая функиия. Таким образом, $u=$ const согласно принципу максимума Бохнера. В результате получим $\theta=\operatorname{grad} u=0$; поэтому солитон Риччи $(g, \xi, \lambda)$ тривиален.

Следующее следствие теоремы 3.13 доказывается аналогично.

Следствие 4.5. Пусть $(g, \xi, \lambda)$ - солитон Риччи на компактном гладком многообразии $M$. Если для тензора Риччи выполняется неравенство $\operatorname{trace}_{g} L_{\xi} \operatorname{Ric} \geqslant 0$ ( $\left.\leqslant 0\right)$, то солитон Риччи $(g, \xi, \lambda)$ тривиален.

Замечание 4.6. Известно, что каждый стационарный или растягивающийся солитон Риччи на компактном гладком многообразии $M$ тривиален (см., например, [18]). С другой стороны, имеется проблема, сформулированная в [18]: существуют ли специальные условия в размерности $n \geqslant 4$, гарантирующие, что сжимающийся компактный солитон Риччи тривиален? Следующие два следствия дают ответ на этот вопрос.

Доказательство следствия 4.7, вытекающего из теоремы 3.5, очевидно.

Следствие 4.7. Пусть $M-$ связное гладкое многообразие $u(g, \xi, \lambda)$ - солитон Риччи с полной римановой метрикой $g$ и неположительной кривизной Риччи на $M$. Если кинетическая энергия потока, порожденного векторным полем $\xi$, бесконечна, то $(g, \xi, \lambda)$ - эйнштейнов солитон Риччи. Кроме того, если оббем многообразия $(M, g)$ бесконечен или кривизна Риччи отрицательна в некоторой точке, то $(g, \xi, \lambda)$ - тривиальный солитон Риччи. 
Из (3.8) и (4.2) заключаем, что дивергенция вектора ускорения объемного расширения $X=$ $(\operatorname{div} \xi) \xi$ имеет вид

$$
\operatorname{div} X=-L_{\xi} s+(s+n \lambda)^{2} .
$$

Кроме того, если $(M, g)$ - полное и ориентированное риманово многообразие, для которого $\|(\operatorname{div} \xi) \xi\| \in L^{1}(M, g)$ и $L_{\xi} s \leqslant 0$, то из (4.3) получаем, что $\operatorname{div} X \geqslant 0$. Тогда согласно обобщенной теореме Грина (см. $[11,12])$ имеем $\operatorname{div} X=0$. Это означает, что $L_{\xi} s=0$ и

$$
s=-n \lambda .
$$

В этом случае из известного тождества Шура $\delta$ Ric $=-2^{-1} \nabla s$ получаем уравнение $\delta$ Ric $=0$. (Напомним, что имеет место равенство $\delta \tilde{\Delta}=\tilde{\Delta} \delta$.) Следовательно, применив оператор дивергенции $\delta$ к обеим частям уравнения

$$
\tilde{\Delta} \theta=2 \operatorname{Ric}(\xi, \cdot)
$$

получим

$$
\operatorname{trace}_{g}(S \cdot \nabla \xi)=0 .
$$

В этом случае из уравнения (4.1) и равенства (4.4) вытекает соотношение $\|$ Ric $\|^{2}=n^{-1} s^{2}$. Следовательно, квадрат бесследовой части тензора Риччи равен нулю, т.е.

$$
\left\|\operatorname{Ric}-n^{-1} s g\right\|^{2}=\|\operatorname{Ric}\|^{2}-n^{-1} s^{2}=0 .
$$

Итак, Ric $=n^{-1} s \cdot g$ и $L_{\xi} g=0$. Это означает, что $\xi$-инфинитезимальная изометрия и $(g, \xi, \lambda)-$ эйнштейнов солитон Риччи. Таким образом, доказано следующее утверждение.

Теорема 4.8. Пусть $(g, \xi, \lambda)$ - солитон Риччи с полной римановой метрикой $g$ на связном ориентированном гладком многообразии $M$, удовлетворяющий следующим условиям:

(i) элемент объема $d \mathrm{Vol}_{g}$ (соответственно, скалярная кривизна $s$ метрики g) является неубывающей (соответственно, невозрастающей) функиией вдоль траекторий потока с вектором скорости $\xi$

(ii) логарифмическая скорость объемного расширения $X=(\operatorname{div} \xi) \xi$ такова, что $\|X\| \in L^{1}(M, g)$. Тогда поток, порожденный полем $\xi$, состоит из изометрических преобразований и $(g, \xi, \lambda)-$ эйнштейнов солитон Риччи.

Замечание 4.9. Теорема 4.8 является обобщением одного из основных результатов работы [13] - теоремы 5.4 .

В частности, при $s=$ const получаем следующее утверждение.

Следствие 4.10. Пусть $(g, \xi, \lambda)$ - солитон Риччи с полной римановой метрикой $g$ постоянной скалярной кривизны на связном ориентированном гладком многообразии $M$, причем логарифмическая скорость обгемного расширения $X=(\operatorname{div} \xi) \xi$ такова, что $\|X\| \in L^{1}(M, g)$. Тогда поток, порожденный полем $\xi$, состоит из изометрических преобразований $u(g, \xi, \lambda)$ - эйнштейнов солитон Риччи.

Замечание 4.11. Следствие 4.10 дополняет результаты статьи [19], в которой изучались полные градиентные солитоны Риччи, имеющие постоянную скалярную кривизну.

Пусть $(g, \xi, \lambda)$ - сжимающийся солитон Риччи с полной метрикой $g$ на связном ориентированном гладком многообразии $M$ и векторное поле $\xi$ удовлетворяет теореме 4.8. Тогда из теоремы 4.1 получаем следующее неравенство: Ric $=n^{-1} s \cdot g>0$, где $s$ - положительная постоянная. Тогда из оценки диаметра Майерса следует, что многообразие $(M, g)$ компактно (см., например, $[27$, с. 251,386$])$. Предположим, что $\operatorname{dim} M=3$; тогда $(M, g)$ имеет постоянную секционную кривизну; в частности, если $M$ односвязнно, то оно изометрично евклидовой сфере $S^{3}$. Для этого случая получаем следующее утверждение. 
Следствие 4.12. Пусть $(g, \xi, \lambda)$ - сжимающийся солитон Риччи с полной римановой метрикой $g$ на связном ориентированном односвязном трехмерном гладком многообразии $M$, причем элемент объема $d \mathrm{Vol}_{g}$ является неубывающей функцией вдоль траекторий потока с вектором скорости $\xi$, а логарифмическая скорость обвемного расширения $X=(\operatorname{div} \xi) \xi$ такова, что $\|X\| \in L^{1}(M, g)$. Тогда многообразие $(M, g)$ изометрично евклидовой сфере $S^{3}$.

Из следствия 3.7 вытекает следующее утверждение.

Следствие 4.13. Пусть $(g, \xi, \lambda)$ - солитон Риччи с полной метрикой $g$, неположительной кривизной Риччи и неприводимой группой голономии $\operatorname{Hol}(g)$ на связном некомпактном гладком многообразии М. Если кинетическая энергия потока, порожденного векторным полем $\xi$, бесконечна, то $(g, \xi, \lambda)$ - тривиальный солитон Риччи.

5. Эволюционные уравнения для скалярной кривизны и тензора Риччи. Рассмотрим однопараметрическое семейство метрик $g(t)$ на многообразии $M$, заданное на временно́м интервале $J \subset \mathbb{R}$. Уравнение Гамильтона для потока Риччи имеет вид

$$
\frac{\partial}{\partial t} g(t)=-2 \operatorname{Ric}(t)
$$

где $\operatorname{Ric}(t)$ - тензор Риччи метрики $g=g(t)$. Известно, что для любой $C^{\infty}$-метрики $g$ на компактном многообразии $M$ существует единственное решение $g(t), t \in[0, \varepsilon)$, уравнения потока Риччи с начальным условием $g(0)=g$ при некотором $\varepsilon>0$ (см. [22]).

Замечание 5.1. Компактные солитоны Риччи являются неподвижными точками потока Риччи (5.1), спроектированного из пространства метрик на его факторпространство по модулю диффеоморфизмов и растяжений и часто возникают как «blow-up»-пределы потоков Риччи на компактных многообразиях.

При заданном потоке Риччи имеем следующее эволюиионное уравнение для скалярной кривизны $s=s(t)$ (см. [15, с. 99]):

$$
\frac{\partial}{\partial t} s(t)=\Delta_{g(t)} s(t)+2\|\operatorname{Ric}(t)\|^{2} .
$$

В предположении, что $\partial s(t) / \partial t \leqslant 0$ для всех $t \in J$, получим из (5.2) неравенство

$$
\Delta_{g(t)} s(t) \leqslant-2\|\operatorname{Ric}(t)\|^{2} \leqslant 0 .
$$

В случае компактного многообразия $M$ получим $\Delta_{g(t)} s(t)=0$, и согласно принципу максимума Бохнера $s=$ const. Тогда из (5.3) заключаем, что $\operatorname{Ric}(t) \equiv 0$. Уравнение (5.2) можно переписать в виде $\partial g(t) / \partial t \equiv 0$; следовательно, поток Риччи (5.1) тривиален. Теперь можем сформулировать следующее очевидное предложение.

Предложение 5.2. Пусть $M$-компактное гладкое многообразие с гамильтоновым потоком Риччи $\partial g(t) / \partial t=-2 \operatorname{Ric}(t)$, где $g(t)$ - однопараметрическое семейство метрик на $M$, определенное на интервале $t \in J \subset \mathbb{R}$. Если $\partial s(t) / \partial t \leqslant 0$ для всех $t \in J$, то поток Риччи тривиален.

В частности, из предложения 5.2 следует, что при нетривиальном потоке Риччи скалярная кривизна $s=s(t)$ не может быть монотонно убывающей функцией. Минимум скалярной кривизны возрастает с течением времени следующим образом (см. [21]):

$$
s_{\min }(t) \geqslant \frac{s_{\min }(0)}{1-\frac{2}{3} t s_{\min }(0)} .
$$

Замечание 5.3. Следствие 4.4 автоматически вытекает из предложения 5.2.

С другой стороны, эволюционное уравнение для тензора Риччи под действием потока Риччи имеет вид

$$
\frac{\partial}{\partial t} \operatorname{Ric}(t)=\Delta_{L} \operatorname{Ric}(t)
$$


(см. $\left[15\right.$, с. 112]), где $\Delta_{L}$ - лапласиан Лихнеровича, определенный метрикой $g(t)$ (см. [15, с. 109]). Из (5.4) получим

$$
\operatorname{trace}_{g(t)} \frac{\partial}{\partial t} \operatorname{Ric}(t)=\Delta_{g(t)} s(t)
$$

В предположении, что

$$
\operatorname{trace}_{g(t)} \frac{\partial}{\partial t} \operatorname{Ric}(t) \geqslant 0 \quad \text { или } \operatorname{trace}_{g(t)} \frac{\partial}{\partial t} \operatorname{Ric}(t) \leqslant 0 \quad \forall t \in J,
$$

из (5.5) получим $\Delta_{g(t)} s(t) \leqslant 0$ или $\Delta_{g(t)} s(t) \geqslant 0$, соответственно. В случае компактного многообразия $M$ при помощи принципа максимума Бохнера получим $s=$ const. В этом случае поток Риччи (5.1) тривиален. Теперь можем сформулировать следующее очевидное предложение.

Предложение 5.4. Пусть $M$-компактное гладкое многообразие с гамильтоновым потоком Риччи $\partial g(t) / \partial t=-2 \operatorname{Ric}(t)$, где $g(t)$ - однопараметрическое семейство метрик на $M$, определенное на временно́м промежутке $J \subset \mathbb{R}$. Если выполняется условие (5.6), то поток Риччи тривиален.

Замечание 5.5. Следствие 4.5 автоматически вытекает из предложения 5.4.

\section{СПИСОК ЛИТЕРАТУРЫ}

1. Арнольд В. И., Хесин Б. А. Топологические методы в гидродинамике. - М.: МЦНМО, 2007.

2. Кобалси Ш. Группы преобразований в дифференциальной геометрии. - М.: Наука, 1986.

3. Степанов C. Е., Цыганок И. И. Инфинитезимальные гармонические преобразования и солитоны Риччи на полных римановых многообразиях// Изв. вузов. Мат. - 2010. - № 3. - С. 97-101.

4. Степанов С. Е., Цыганок И. И. Гармонические преобразования полного риманова многообразия// Мат. заметки. - 2016. - 100, № 3. - С. 441-449.

5. Степанов С. Е., Шандра И. Г. Гармонические диффеоморфизмы многообразий// Алгебра и анализ. -2004 . -16 , № 2. - C. 154-171.

6. Степанов С. Е., Шандра И. Г. Новые характеристики инфинитезимальной изометрии и тривиального солитона Риччи// Мат. заметки. - 2012. - 92, № 3. - С. 459-462.

7. Степанов С. Е., Шелепова В. Н. Заметка о солитонах Риччи// Мат. заметки. - 2009. - 86, № 3. C. $474-477$.

8. Bérard P. H. From vanishing theorems to estimating theorems: the Bochner technique revisited// Bull. Am. Math. Soc. - 1988. - 19, № 2. - P. 371-406.

9. Bochner S., Yano K. Curvature and Betti Numbers. — Princeton: Princeton Univ. Press, 1953.

10. Calabi E. An extension of E. Hopf's maximum principle with an application to Riemannian geometry// Duke Math. J. - 1957. - 25. - P. 45-56.

11. Caminha A. The geometry of closed conformal vector fields on Riemannian spaces// Bull. Braz. Math. Soc. New Ser. - 2011. - 42, № 2. - P. 277-300.

12. Caminha A., Souza P., Camargo F. Complete foliations of space forms by hypersurfaces// Bull. Braz. Math. Soc. New Ser. - 2010. - 41, № 3. - P. 339-353.

13. Catino G., Mastrolia P., Monticelli D., Rigoli M. Analytical and geometric properties of generic Ricci solitons// Trans. Am. Math. Soc. — 2016. - 368, № 11. - P. 7533-7549.

14. Chow B., Knopf D. The Ricci Flow: An Introduction. - Providence, Rhode Island: Am. Math. Soc., 2004.

15. Chow B., Lu P., Ni L. Hamilton's Ricci Flow. - Providence, Rhode Island: Am. Math. Soc., 2006.

16. Davis $W$. R., Oliver D. R. Matter field space times admitting symmetry mappings satisfying vanishing contraction of the Lie deformation of the Ricci tensor// Ann. Inst. H. Poincaré, Sec. A (N.S.). - 1978. 28, № 2. - P. 197-206.

17. Eells J., Sampson J. H. Harmonic mappings of Riemannian manifolds// Am. J. Math. — 1964. — 86, № 1. - P. 109-160.

18. Eminenti M., La Nave G., Mantegazza C. Ricci solitons: The equation point of view// Manuscr. Math. 2008. - 127, № 3. - P. 345-367.

19. Fernandez-Lopez M., Garcia-Rio E. On gradient Ricci solitons with constant scalar curvature// Proc. Am. Math. Soc. - 2016. - 144, № 1. - P. 369-378. 
20. Green L. H., Norris L. K., Oliver D. R., Davis W. R. The Robertson-Walker metric and the symmetries belong to the family of contracted Ricci collineations// Gen. Relat. Gravit. — 1997. — 8, № 9. — P. 731-736.

21. Gromov M. Four Lectures on Scalar Curvature/ arXiv: 1908.10612v3 [math. DG] .

22. Hamilton R. S. Three-manifolds with positive Ricci curvature// J. Diff. Geom. — 1982. — 17, № 2. — P. 255-306.

23. Hélein F., Wood J. Harmonic maps// in: Handbook of Global Analysis. — Amsterdam: Elsevier, 2008. P. 417-491.

24. Li P., Schoen R. $L^{p}$ and mean value properties of subharmonic functions on Riemannian manifolds// Acta Math. - 1984. - 153. - P. 279-301.

25. Nouhaud O. Transformations infinitésimales harmoniques// C. R. Acad. Sci. Paris. — 1972. — 274. — P. 573-576.

26. O'Neil B. Semi-Riemannian Geometry with Applications to Relativity. — San Diego: Academic Press, 1983.

27. Petersen P. Riemannian Geometry. - Berlin: Springer-Verlag, 2016.

28. Pigola S., Rigoli M., Setti A. G. Vanishing and Finiteness Results in Geometric Analysis. A Generalization of the Bochner Technique. - Berlin: Birkhäuser, 2008.

29. Schoen R., Yau S. T. Harmonic maps and the topology of stable hypersurfaces and smooth manifolds with nonnegative Ricci curvature// Comment. Math. Helv. — 1976. — 61, № 1. — P. 333-341.

30. Stepanov S. E., Mikeš J. The spectral theory of the Yano Laplacian with some of its applications// Ann. Glob. Anal. Geom. - 2015. - 48. - P. 37-46.

31. Stepanov S. E., Shandra I. G. Geometry of infinitesimal harmonic transformation// Ann. Glob. Anal. Geom. - 2003. - 24. - P. 291-297.

32. Stepanov S. E., Tsyganok I. I. Vanishing theorems for harmonic mappings into nonnegativity curved smooth manifolds and their applications// Manuscr. Math. — 2017. — 154, № 1-2. — P. 79-90.

33. Stepanov S. E., Tsyganok I. I., Mikěs J. From infinitesimal harmonic transformations to Ricci solitons// Math. Bohem. - 2013. - 1. - P. 25-36.

34. Wu H. A remark on the Bochner technique in differential geometry// Proc. Am. Math. Soc. — 1980. — 78, № 3. - P. 403-408.

35. Xin Y. Geometry of Harmonic Maps. - Boston-Berlin: Springer-Verlag, 2012.

36. Yau S. T. Some function-theoretic properties of complete Riemannian manifold and their applications to geometry// Indiana Univ. Math. J. — 1976. — 25, № 7. — P. 659-679.

37. Yau S. T. Erratum: Some function-theoretic properties of complete Riemannian manifold and their applications to geometry// Indiana Univ. Math. J. — 1982. — 31, № 4. - P. 607.

Александрова Ирина Александровна

Финансовый университет при Правительстве Российской Федерации

E-mail: IAleksandrova@fa.ru

Степанов Сергей Евгеньевич

Финансовый университет при Правительстве Российской Федерации;

Всероссийский институт научной и технической информации

Российской академии наук (ВИНИТИ РАН)

E-mail: s.e.stepanov@mail.ru

Цыганок Ирина Ивановна

Финансовый университет при Правительстве Российской Федерации

E-mail: i.i.tsyganok@mail.ru 